\title{
A Novel Combination Forecasting Algorithm Based on Time Series
}

\author{
Lihua Yang, Baolin Li, Xuetao Li and Lvjiang Yin \\ School of Economics and Management,Hubei University of Automotive \\ Technology, Shiyan, 442002, China \\ yanglh168@126.com;libaolin108@163.com; 857890008@qq.com; \\ 568235892@qq.com
}

\begin{abstract}
To effectively predict cigarette sales and improve the competitiveness of tobacco business enterprises, the characteristics of actual cigarette sales were detailed analyzed. Due to the long-term growth trends, seasonal fluctuations and the nonlinearity of monthly sales, we established three single forecasting models, which are Exponential Smoothing (ES), Seasonal Decomposition (SD) and Radial Basis Function (RBF) neural network. After obtaining the predicted value of three single models, the combination forecasting model was proposed. The weights of the three single models were computed using Mean Absolute Error and the mean relative error respectively, the result shows that relative error is more effective. A dynamic weight combination forecasting method based on $R B F$ is proposed and compared with fixed weight method. Finally, the prediction accuracy of different models was compared based on the criteria of MAPE and RMSE, and the effectiveness of the combination method was proved, the proposed model can take advantage of the strengths of the three single models, the results indicate that the combination forecasting model suitable for cigarette sales has higher prediction accuracy. In some cases, the prediction accuracy of the fixed weight combination model is better than the dynamic weight combination model. The results can provide a certain reference to cigarette sales forecasting.
\end{abstract}

Keywords: combination forecasting, cigarette sales, RBF, dynamic weight

\section{Introduction}

China is the world's largest producer and consumer of tobacco, with the rapid development of china's economy, which plays an important role on the national economy and structure stability. In recent years, the policy of "organizing supply according to customer orders" is being promoted broadly and thoroughly in Chinese tobacco industry. As a monopoly market, how to predict the market demand accurately so as to provide a real and efficient reference for the industry is particularly important in the background of the separation of industrial and commercial enterprises. Therefore, the sales forecasting accuracy has become a hot spot for tobacco commercial industry, which can help enterprise to improve the competitiveness of marketing, satisfy customer demand and improve the service level.

In recent years, many scholars have researched the sales forecasting methods. In generally, the forecasting methods can be generally divided into two categories, namely, the qualitative forecasting methods and quantitative forecasting methods. The latter methods include some traditional statistics methods, such as moving average, exponential smoothing, seasonal decomposition and multiple regression analysis [1]. Time series forecasting, as an important tool in many decision support systems, has been extensively studied and applied for sales forecasting over the past few decades. The application of these traditional forecasting methods has specific requirement for the dataset, however, 
in practical application, the time series of actual sales can't well satisfy the needs of the models, thus the forecasting effect will be more or less affected.

In fact, cigarette sales are affected by many complex factors, such as economic situation, state policy, the income, and so on. These complicated factors cause the remarkable fluctuation and non-linear characteristics of the historic sales data, some intelligent algorithms are applied to solve the problems. ANN models have been revealed to be more efficient and effective than many traditional statistical forecasting models. Despite the reported advantages, it is relatively more time-consuming for ANN to perform forecasting, a new model which employs both the extreme learning machine and the traditional statistical methods is proposed, a comparison with other traditional methods has shown that this ELM fast forecasting model is quick and effective [2]. Support vector regression was applied to the newspaper or magazines sales forecasting is a superior method [3].

The above methods can find out different characteristics of sales forecasting. However, the single model has its own limitation, which can only use some certain effective information. Therefore, the combination forecasting model was proposed by Bates and Granger in 1969[4], they systematic studied the combination forecasting model for the first time. Combination forecasting model can summarize the useful information given by different forecasting methods, so it can overcome the limitations of individual models and gather information more effectively. After that, combination forecasting model has gained many attentions. To improve the accuracy of fashion retail forecasting, a two-stage dynamic forecasting model is proposed, which is combined with both long-term and short-term predictions [5]. A hybrid forecasting scheme which combines the classic SARIMA method and wavelet transform is proposed [6]. Some researchers concluded that the result of combination forecast must not be superior to the best individual model, they were skeptical about the effectiveness of the combination models [7]. But in reality, when we don't know which model is the best, the choice of combination model can mostly lead to better performance than the individual model. So the majority are supportive attitude.

The core points of combination forecast are the selection of individual model and the calculation of weight. The selection of individual model should be based on the actual data characteristics and experiments. At present, the calculation methods about the combination weights can be divided into two categories: fixed weight and dynamic weight. Fixed weight is permanent, the weight at each point is the same, and dynamic weight is time-variant. The former has a longer history and more mature technique, while the latter is still in the exploratory stage [8]. Some researchers believe that the precision of the time-variant weight method is much higher than permanent weight [9]. A new predicting model based on neural network of variable weighting modulus was put forward to forecast the working conditions, the core idea of the new method is to determine the weight dynamically, using this new method, the predicting precision is improved [10], but BP neural network requires a large number of samples for training and exists the local minimum problem. A changeable weight combination model was established using regression method to calculate the weight coefficient [11], but the application of regression method requires lots of samples and the weight coefficient in the time series has the certain regularity.

A few studies have been proposed to examine the accuracy of combination model for cigarette sales. A new market forecasting system for tobacco wholesalers in China based on a new developed market demand forecasting model is presented, this model can help sales company to improve the forecasting accuracy for annual and monthly market demands significantly [12]. Using the combination forecasting method which based on PLS to predict the province's cigarette sales of the next year, the results show that the prediction accuracy is good, which could provide a certain reference to... cigarette sales forecasting in a province[13]. A combination forecasting method based on time series 
method and PLS was established to predict the cigarette sales of the next year [14], the results show that the accuracy of prediction is good.

However, intelligent algorithm was not applied in the above cigarette sales forecasting model. Consider the complexity of actual data, the intelligent algorithm of RBF is introduced. A novel combination model is established to improve accuracy of sales forecasting, in which three forecasting models based on Exponential Smoothing (ES), Seasonal Decomposition (SD) and Radial Basis Function (RBF) neural network are introduced. Actual monthly sales data from January 2008 to June 2013 are used to establish the model, actual monthly sales data from July 2013 to December 2013 are used to test the model. These models are compared based on the evaluation criterions of MAPE and RMSE between predicted values and true ones. This new forecasting model is an extension to the combination prediction method. The calculation of fixed weight coefficients on the basis of relative error and absolute error was explored, result shows that fixed weight coefficient using relative error is more accuracy. The fixed weight coefficients using relative error and the dynamic weight coefficients using RBF were compared, the experiment indicates that the dynamic weight coefficient is not always better than fixed weight coefficient in the aspect of improving precision.

The paper is organized as follows. Firstly, we obtain the related cigarette sales data, analyzing the data characteristics. Secondly, three single models were established based on the selection data. Thirdly, the weights of combination model were calculated based on relative error and absolute error respectively, by comparing the predictive accuracy of the different combination models, the relative error is more effective in computing the weights. Fourthly, the dynamic weight method based on RBF neural network was established, and compared with the fixed weight method. Finally, the forecasting results of four models were compared based on the criteria of MAPE and RMSE, then the optimization model was proposed.

\section{Data Collection}

There are three kinds of prediction type in cigarette commercial enterprise, which are monthly forecast, semi-annual forecast and annual forecast. The object of annual forecast is all brands' yearly total sales. Annual forecast is based on analysis of historical sales data and influencing factors such as marketing policy, regional consumption structure and economic conditions. The accuracy of annual forecast is based on the quantitative methods and qualitative methods of human experience. Semi-annual forecast is based on annual forecast results and the quarterly influencing factors. Monthly forecast includes market forecast and brand forecast, the results of monthly forecast are foundation to monthly supply and demand planning between industrial and commercial industries, which are important to marketing management, inventory management and logistic delivery management.

In this paper, we obtained monthly sales data from cigarette commercial enterprise. To do monthly sales forecasting, the models are constructed based on monthly sales data from January 2008 to June 2013, a total of 66 samples. The forecasting models are tested using the monthly sales data, from July 2013 to December 2013, a total of 6 samples. The mothly sales data are shown in Figure 1. 


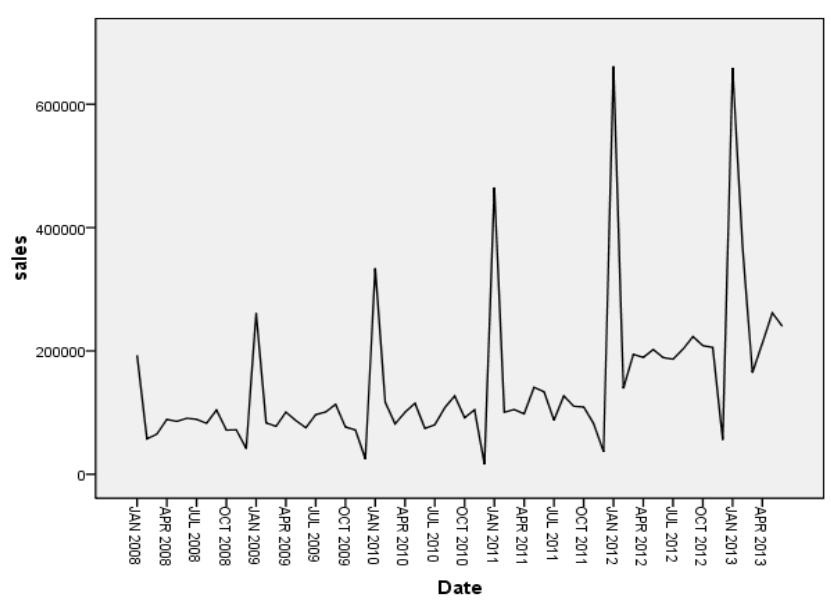

Figure 1. Sequence Chart of Monthly Sales

From the Figure 1, we can see that the time series appear long-term growth trends and obviously seasonal fluctuations. There is an obvious seasonal growth between January and February in every year. Meanwhile, there is a seasonal decrease between November and January. The sequence chart indicates that seasonal underlying periodicity probably is 12 months. We can also notice that the seasonal variations appear to grow with the upward series trend, which implies a multiplicative model is better than an additive model.

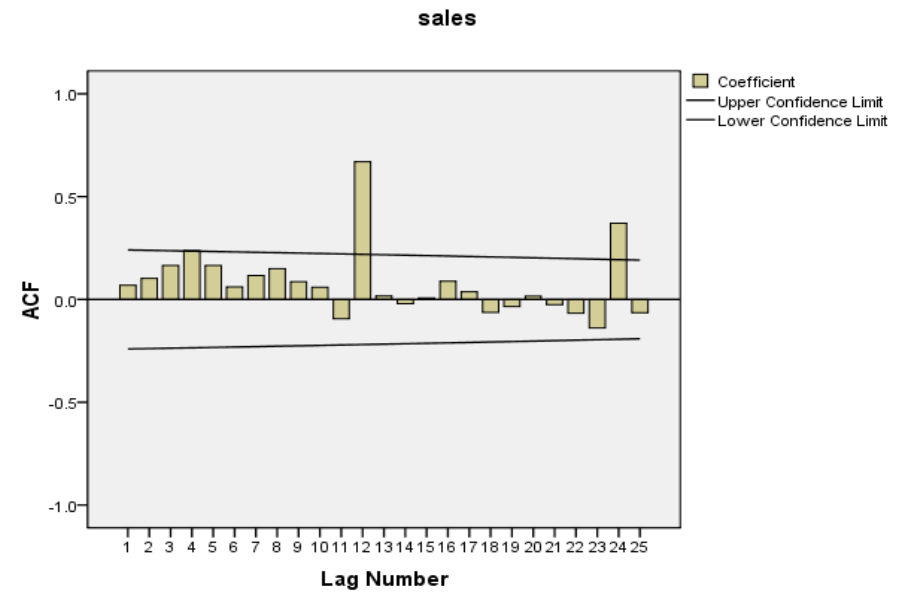

Figure 2. Autocorrelation Analysis

The figure of autocorrelation function shows the complexity of the series. We can gains a more quantitative conclusion about the underlying periodicity from the autocorrelations of the time series, the autocorrelation function in Figure 2 shows a significant peak at a lag of 12 and a lag of 24 , which exceed the confidence interval. The figure suggests the presence of an annual seasonal component in the data. It is a periodic non-stationary time series.

\section{Forecasting Results of Three Single Models}

\subsection{Exponential Smoothing Model}

Exponential smoothing was first proposed by Robert Goodell Brown in 1956, and then expanded by Charles C. Holt in 1957.Exponential smoothing is a technique 
that can be applied to time series data, either to produce smoothed data for presentation, or to make forecasts. The past observations are weighted equally in the simple moving average, but exponential smoothing assigns exponentially decreasing weights over time. Exponential smoothing models are classified as either seasonal or non-seasonal. The simplest form of exponential smoothing is appropriate for series in which there is no trend or seasonality. Expression is as follows:

$$
s_{t}=\alpha x_{t-1}+(1-\alpha) s_{t-1}
$$

Where $\alpha$ is the smoothing factor, and $0<\alpha<1$. The smoothed statistic ${ }^{s_{t}}$ is a simple weighted average of the previous observation $x_{t-1}$ and the previous smoothed statistic ${ }^{{ }_{t-1}}$. Simple exponential smoothing is easily applied, and it can also be classified as an autoregressive integrated moving average (ARIMA) $(0,1,1)$ model with no constant term.

Winters' additive model is appropriate for series with a linear trend and a seasonal effect that does not depend on the level of the series. Winters' additive exponential smoothing is most similar to an ARIMA model with zero orders of auto-regression, one order of differencing, one order of seasonal differencing, and $p$ +1 orders of moving average, where $p$ is the number of periods in a seasonal interval. Winters' multiplicative model is appropriate for series with a linear trend and a seasonal effect that depends on the level of the series. Its smoothing parameters are level, trend, and season. Because there are both the characteristics of trend and seasonal fluctuations in the actual cigarette sales data, therefore, we selected the winters' additive model to forecast sales by comparing them. Though the fitting capacity of winters' multiplicative model is better than that of winters' additive model, the forecasting accuracy of winters' multiplicative model is worse than that of winters' additive model. So the multiplicative model is applied.

Through experiment, stationary R-squared obtained is 0.513 , which means that the model under consideration is better than simple mean model. $\mathrm{R}$-squared is 0.861 , which represents $86 \%$ of the total variation in the series can be explained by this model. These mean the fitting capacities of models are acceptable.

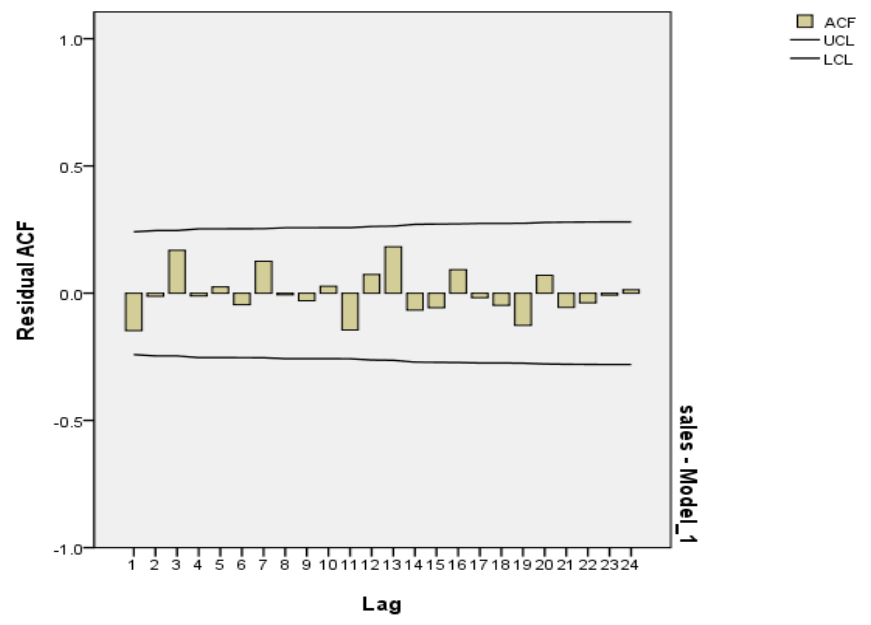

Figure 3. Autocorrelation Analysis of Residual Series

We can examine the autocorrelations and partial autocorrelations of the time series to determine the underlying periodicity. From the Figure 3, we can see that the autocorrelation coefficient of residuals are near zero which indicate that the residual 
series is white noise series, and this exponential smoothing model is acceptable to forecast cigarette sales.

\section{2. Seasonal Decomposition Model}

An observed time series can be decomposed into four components: the long term trend (T), the seasonal component (S), the cyclical component (C) and the irregular component (I). Trend component refers to overall upward or downward movement over a period of years. Seasonal component is upward or downward swings observed within 1 year. Cyclical component usually is irregular in depth and duration and often correspond to periods of economic expansion or contraction. Irregular component is also known as the residual, which is what remains after the seasonal and trend components of a time series have been removed. Seasonal decomposition model can be divided into two models according to the relations between the four components, multiplicative model and additive model. The formula of additive model is $Y=T+S+C+I$, this is the most appropriate model for series where the magnitude of the seasonal component does not appear to be affected by the level of the series. The formula of multiplicative mode is $Y=T S C I$, this is the most frequently used model for time series where the magnitude of the seasonal fluctuations is proportional to the level of the series.

From the above analysis, we can draw the conclusion that multiplicative seasonal adjustment model can be applied to cigarette sales forecasting. The seasonal decomposition procedure can creates four new variables by analyzing the original variables, where SAF is seasonal adjustment factors, representing seasonal variation; SAS is seasonally adjusted series, representing the original series removing seasonal variations; STC is smoothed trend-cycle component, which is a smoothed series that shows both trend and cyclic components; ERR is the residual component of the series.

To perform a trend analysis, we should firstly remove any seasonal variations present in the data. The seasonal component of a periodic time series can be removed easily using the seasonal decomposition procedure. Here, the moving average weight is endpoints weighted by 0.5 .

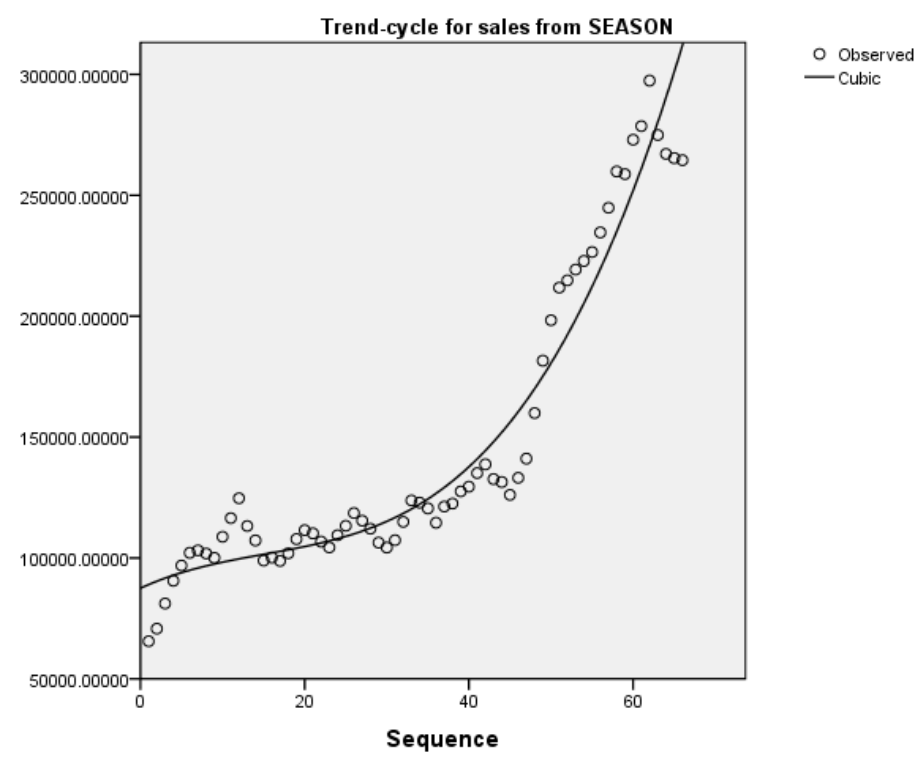

Figure 4. The Smoothed Trend-Cycle Series

The smoothed trend-cycle series show a clear upward trend in the following Figure 4. Curve estimation is applied because the relationship between the dependent variable and 
the independent variable is nonlinear. Curve fitting function is cubic. R-squared is 0.938 , the model can be acceptable.

\subsection{RBF Model}

RBF neural network is an artificial neural network that uses radial basis functions as activation functions, which has been widely used in many fields, such as pattern recognition, data classification, non-linear modeling, system modeling, and fault diagnosis. It is better than Back Propagation (BP) neural network in approximation capability, fault tolerance, and adaptation. The structure of RBF commonly consists of three layers, namely, input layer, hidden layer with a non-linear RBF activation function and a linear output layer, and each layer contains a number of neurons. Input layer nodes transfer signals to the hidden layer, then the hidden layer performs a fixed nonlinear transformation with no adjustable parameters, and the output node is usually a simple linear function. This structure is also known as feed-forward architecture because the connections in the network flow forward from the input layer to the output layer without any feedback loops. An RBF network with enough hidden neurons can approximate any continuous function with arbitrary precision. In addition to the unnormalized architecture, RBF networks can also be normalized.

From the above analysis, we can see that seasonal periodicity of the cigarette sales is 12 months. Monthly sales data from January 2008 to December 2008 are selected as input nodes, monthly sales data of January 2009 is selected as corresponding output node, and these data constitute the first record in the dataset. Similarly, the variable of nextmonth is selected as the dependent variable, and monthly sales of 12 months before nextmonth are selected as the independent variables. Therefore, the training dataset consists of 54 records, monthly sales data from July 2013 to December 2013 are assigned to the holdout sample. The holdout sample is another independent set of data records used to assess the final neural network; the error for the holdout sample gives an "honest" estimate of the predictive ability of the model because the holdout cases were not used to build the model. The best number of hidden units is automatically computed according to the smallest error in the training dataset, the activation function for the hidden layer is the ordinary radial basis function, and activation function for the output layer is identity function.

The processing results show that 54 cases were assigned to the training data set, 6 cases to the holdout data set. There are 12 neurons in the input layer according to the number of independent variables, likewise, there is 1 unit in the output layer, and there are 2 units in the hidden layer.

\subsection{Results Comparison of Three Single Models}

The predictive results of the three single models are shown in Table 1.

Table 1. The Forecasting Results of Three Single Models

\begin{tabular}{ccccr}
\hline Date & Sales & ES & SD & RBF \\
\hline 201307 & 257623 & 231021 & 266782 & 214 \\
& & & & 807 \\
201308 & 248101 & 245466 & 293622 & 214 \\
& & & & 894 \\
201309 & 263272 & 261314 & 349229 & 214 \\
& & & & 936 \\
201310 & \multirow{2}{*}{301058} & 243690 & 263065 & 214 \\
& & & & 981 \\
201311 & \multirow{2}{*}{303427} & 236511 & 278746 & 215 \\
& & & & 068 \\
201312 & \multirow{2}{*}{183517} & \multirow{2}{*}{92804} & 84845 & 215 \\
& & & & \\
\hline
\end{tabular}


Where the measuring unit of cigarette sales is strip, sales stands for actual sales, ES stands for the predicted values of Exponential Smoothing model, SD stands for the predicted values of Seasonal Decomposition model, RBF stands for the predicted values of RBF neural network model.

From the table 1, we can see that the forecasting results of three single models were different, because the single model can take advantage of different information of the data.

In this paper, the MAPE and RMSE are regarded as the criteria for comparing the forecasting precision of different models. The related formulae are expressed as follows:

$$
\begin{aligned}
& \text { MAPE }=\frac{100}{n} \sum_{t=1}^{n}\left|\frac{\overline{y_{t}}-y_{t}}{y_{t}}\right| \\
& R M S E=\sqrt{\frac{1}{n} \sum_{t=1}^{n}\left(\overline{y_{t}}-y_{t}\right)^{2}}
\end{aligned}
$$

Where $\bar{y}_{t}$ represents the predicted value at the t-th period, ${ }^{y_{t}}$ represents the actual value at the $\mathrm{t}$-th period, $\mathrm{n}$ is the total number of observations, where $\mathrm{n}$ is equal to 6 .

The results are shown in Table 2.

Table 2. The Comparison of Three Single Models

\begin{tabular}{lcrr}
\hline Criteria & ES & SD & RBF \\
\hline MAPE & 17.11 & 21.51 & 20.55 \\
RMSE & 52782.77 & 59628.64 & 59844.71 \\
\hline
\end{tabular}

According to the criteria of MAPE and RMSE, the forecasting results of ES model are the best of three single models, the precision of RBF model is better than that of SD model based on the criterion of MAPE, however, the precision of RBF model is worse than that of SD model based on the criterion of RMSE.

\section{Combination Forecasting}

Different forecasting models based on the same dataset may obtain different forecasting results. Some useful information will be discarded if we simply abandon some models due to the bigger error. To solve the problem, combination model was firstly proposed by Bates and Granger in 1969. After that, the study of combination forecasting has become the hot topic in the forecasting field. The combination model can reduce the influence of the single model by random factors, which can improve the accuracy and stability of forecasting. How to assign appropriate weight to single model is very important in the combination forecasting model, the reasonable weight will greatly improve predicted accuracy. Calculation of the weight can be divided into two categories, fixed weight calculation and dynamic weight calculation.

\subsection{Fixed Weight Calculation}

There are some methods to calculate fixed weight, such as arithmetic average method, inverse variance method, mean square inverse method, simple additive weighting method, and standard deviation method. The combination forecasting model formula can be expressed as follows:

$$
Y=W_{1} Y_{1}+W_{2} Y_{2}+W_{3} Y_{3}
$$


Where ${ }^{Y}$ is the predictive value of combination forecasting model, $Y_{1}$ is the predictive value of Exponential Smoothing model, $Y_{2}$ is the predictive value of Seasonal Decomposition model, ${ }^{Y_{3}}$ is the predictive value of RBF neural network. ${ }^{W_{i}}$ is the weight assigned to the $\mathrm{i}$-th model. In the combination model, the sum of the weights is constrained to be equal to one, and the value of the weight cannot be less than zero.

Here, we choose inverse variance method to calculate the weight. Its principle is that the model of the smaller sum of square error should have a higher weight, and the model of the higher sum of square error should have a smaller weight. Expressed in a formula, it can be written as follows.

$$
W_{i}=D_{i}^{-1} / \sum_{i=1}^{m} D_{i}^{-1}, i=1,2, \cdots, m
$$

Where $\mathrm{m}$ is the number of model, ${ }^{W_{i}}$ is the weight of $\mathrm{i}$-th model, ${ }^{D_{i}}$ represents the sum of square error of $\mathrm{i}$-th model, the formula is as follows.

$$
D_{i}=\sum_{t=1}^{n}\left(y_{t}-\overline{y_{t}}\right)^{2}
$$

Where $y_{t}$ denotes the predicted value at the $\mathrm{t}$-th period, ${ }_{t}$ denotes the actual value at the t-th period.

When we know the predicted values of different single forecasting models, we can compute the corresponding weight coefficient, then multiplying the predictive value of single model, finally the combination forecasting results can be obtained.

At present, the combination forecasting model is generally established based on the criteria of the sum of square error, but some researchers point out that the relative error may be more accurate than the absolute error [15]. Considering the forecasting accuracy of relative error, here, the mean relative error is proposed to compute the weight of the single model, the formula is as follows.

$$
D_{i}^{\prime}=\frac{1}{n} \sum_{t=1}^{n}\left|\frac{\overline{y_{t}}-y_{t}}{y_{t}}\right|
$$

In the traditional inverse variance method, ${ }^{D_{i}}$ can be transformed to the following formula.

$$
D_{i}^{\prime \prime}=\frac{1}{n} \sum_{t=1}^{n}\left|\overline{y_{t}}-y_{t}\right|
$$

\begin{tabular}{|c|c|c|}
\hline Model & $\begin{array}{l}\text { Weight coefficient } \\
\text { based on relative } \\
\text { error }\end{array}$ & $\begin{array}{l}\text { Weight coefficient } \\
\text { based on absolute } \\
\text { error }\end{array}$ \\
\hline ES & 0.38 & 0.39 \\
\hline SD & 0.30 & 0.32 \\
\hline RBF & 0.32 & 0.29 \\
\hline
\end{tabular}

Substituting (6) and (7) into (4) yields the corresponding weights, the different weights are shown in Table 3.

Table 3. The Comparison of the Weights

Based on the above weights, we can respectively calculate the forecasting result of different combination models. The first combination model use mean relative error to calculate the weights, the corresponding formula is as follows 


$$
Y^{\prime}=0.38 Y_{1}+0.30 Y_{2}+0.32 Y_{3}
$$

The second combination model use mean absolute error to calculate the weights, the formula is as follows.

$$
Y^{\prime \prime}=0.39 Y_{1}+0.32 Y_{2}+0.29 Y_{3}
$$

The forecasting results of different combination models are shown in Table 4.

Table 4. The Forecasting Results of Different Combination Model

\begin{tabular}{ccccc}
\hline Date & Sales & ES & CM 1 & CM2 \\
\hline 201307 & 257623 & 231021 & 236707 & 237689 \\
201308 & 248101 & 245466 & 250355 & 251902 \\
201309 & 263272 & 261314 & 273229 & 275810 \\
201310 & 301058 & 243690 & 240458 & 241504 \\
201311 & 303427 & 236511 & 242500 & 243719 \\
201312 & 183517 & 92804 & 129152 & 125869 \\
\hline
\end{tabular}

Where CM1 stands for the first combination model, CM2 stands for the second combination model. The forecasting results of ES model are the best of three single models. Therefore, the two combination models are compared with ES model.

The comparison of forecasting results is shown in Table 5.

Table 5. Comparison Results of Combination Models

\begin{tabular}{lrrr}
\hline Criteria & \multicolumn{2}{c}{ ES } & \multicolumn{2}{c}{ CM1 } & \multicolumn{2}{c}{ CM2 } \\
\hline MAPE & 17.11 & 13.77 & 14.15 \\
RMSE & 52782.77 & 42586.45 & 42825.28 \\
\hline
\end{tabular}

From Table 5, we can draw the conclusions that MAPE and RMSE of two combination models are lower than the best ES model, that is, the precision of the combination models is higher than that of three single models in terms of the MAPE and RMSE. Because the combination forecasting model can take into account the more factors and make use of useful information of every single model, so we obtain a more accuracy forecasting result which provide more scientific decision-making. By comparison, the MAPE and RMSE of the CM1 is lower than that of the CM2, the result indicates that relative error is prior to absolute error in the criteria of calculating weight.

\subsection{Dynamic Weight Calculation}

To a single prediction model, fixed weight coefficient at different period is the same. However, single forecasting model may have different prediction accuracy at the same period. Therefore, the dynamic weight is proposed. Some researchers believe that the forecasting precision of dynamic weight coefficients is higher than fixed weight coefficients [8-11]. In this paper, we applied intelligent algorithm of RBF to calculate the dynamic weight. Its idea is to apply the forecasting results of single model as the independent variables, the actual cigarette sales as the dependent variable, then constructing the RBF neural network. The neural network contains 3 input nodes and 1 output node. The activation function for the hidden layer is the ordinary radial basis function, and activation function for the output layer is identity function. 
The comparison results of fixed weight and dynamic weight models are shown in Table6. We can see that MAPE and RMSE of two dynamic weights forecasting models based on RBF algorithm are much higher than that of CM1 and CM2. Therefore, the forecasting precision of dynamic weight coefficients is lower than fixed weight coefficients.

Table 6. Comparison Results of Fixed Weight and Dynamic Weight Models

\begin{tabular}{lrrrr}
\hline Criteria & \multicolumn{1}{l}{ CM1 } & \multicolumn{1}{c}{ CM2 } & \multicolumn{1}{c}{ RBFW } & \multicolumn{1}{c}{ RBFDW2 } \\
\hline MAPE & 13.77 & 14.15 & 28.48 & 35.53 \\
RMSE & 42586.45 & 42825.28 & 82300.47 & 104134.7 \\
\hline
\end{tabular}

RBFDW1 stands for the first dynamic weight combination model, which take the prediction results of the three single models as the input variable. RBFDW2 stands for the second dynamic weight combination model, which also has three input variables, and the formula of input variables is as follows:

$$
V_{i}=\bar{Y}_{t} / e_{t}, i=1,2,3
$$

Where $e_{t}=\left|\bar{Y}_{t}-Y_{t}\right|, \bar{Y}_{t}$ represents the predicted value at the t-th period of i-th single forecasting model. ${ }^{Y_{t}}$ represents the actual value at the t-th period, ${ }^{e_{t}}$ stands for the absolute error. $1 / e_{t}$ stands for the dynamic weight coefficient, which is not fixed value because ${ }^{e_{t}}$ is different at different period. The basic idea is that the model of the smaller absolute error should have a higher weight, and the model of the higher absolute error should have a smaller weight, so the weight coefficient is change according to different samples.

\section{Conclusions}

In this paper, we summarized the achievements of previous studies on combination forecasting model. A novel optimal weight combination forecasting model is proposed, and a new fixed weight calculation method based on mean relative error is also introduced. The main conclusions are described as follows.

1) A novel combination forecasting model based on Exponential Smoothing (ES), Seasonal Decomposition (SD) and Radial Basis Function (RBF) neural network is proposed. The intelligent algorithm of RBF is introduced to predict the monthly cigarette sales, which is an extension to the cigarette sales prediction method. The results indicate that predictive accuracy of combination model is better than that of three single models in terms of the MAPE and RMSE, and ES model is better than the SD and RBF model among the three single models. Different models can achieve different accuracy for forecasting monthly sales due to using the different information from the data.

2) A new fixed weight calculation method called inverse variance method is proposed. The fixed weight coefficients of combination model are calculated respectively according to the mean absolute error and the mean relative error. Experiments indicate that relative error is prior to absolute error in the criteria of calculating weight, because the MAPE and RMSE of combination model based on relative error is both lower than that of absolute error.

3) A new dynamic weight calculation method based on RBF is proposed and realized. Most researchers believe that the prediction accuracy of dynamic weight 
method is higher than fixed weight method. However, experiment results indicate that MAPE and RMSE of two dynamic weights forecasting models based on RBF algorithm are much higher than that of CM1 and CM2. The prediction accuracy of the fixed weight combination model is better than the dynamic weight combination model. When imported the RBF model, it can also increase the complexity, which may lead to over fitting. Therefore, the prediction results are even worse than fixed weight method.

In practice, it's difficult to decide which model is suitable for the actual time series, so a great deal of experiments should be tried to select more appropriate model. Due to the complexity of time series, the combination model usually has better forecasting accuracy due to using the advantages of single model. Our experiment results confirm it. We believe that the novel combination model is highly applicable for forecasting sales in the commercial industry.

\section{Acknowledgements}

This paper is granted by Research Project of Hubei Provincial Department of Education (NO. 2012Y051), and Special Research Foundation of Young Teachers of Hubei University of Automotive Technology (No. 2012XQ02).

\section{References}

[1] P. Yin, Z. J. Wang and D. Y. Xiao, "Prediction of companies' failure risk based on various prediction rough neural networks", Journal of Management Science, vol. 23, no. 4, (2010), pp. 15-26.

[2] Y. Yu, T. M. Choi and C. L. Hui, "An intelligent fast sales forecasting model for fashion products", Expert Systems with Applications, vol. 38, no. 6, (2010), pp. 7373-7379.

[3] X. D. Yu, Z. Q. Qi and Y. M. Zhao, "Support Vector Regression for Newspaper/Magazine Sales Forecasting", Procedia Computer Science, vol. 17, (2013), pp. 1055-1062.

[4] J. M. Bates and C. W. J. Granger, "The combination of Forecasts", Operations Research Quarterly, vol. 20, no. 4, (1969), pp. 451-468.

[5] Y. R. Ni and F. Y. Fan, "A two-stage dynamic sales forecasting model for the fashion retail", Expert Systems with Applications, vol. 38, no. 3, (2010), pp. 1529-1536.

[6] T. M. Choi, Y. Yu and K. F. Au, "A hybrid SARIMA wavelet transform method for sales forecasting", Decision Support Systems, vol. 51,no. 1, (2010), pp. 130-140.

[7] M. Hibon and T. Evgeniou, "To combine or not to combine: selecting among forecasts and their combinations", International Journal of Forecasting, vol. 21, no. 1, (2005), pp.15-24.

[8] G. H. Zhao, S. F. Guo, T. J. Shen, "An investigation of coal demand in China based on the variable weight combination forecasting model", Journal of Resources and Ecology, vol. 2, no. 2, (2011), pp. 126-131.

[9] J. F. Wang, R. Y. Fei and X. L. Xu, "Rotating machinery condition optimization prediction method of variable weight combination RBF model research", Journal of Beijing University of Technology, vol.39, no.1, (2013), pp. 7-13.

[10] X. L. Xu, H. G. Xu and A. D. Cao, "A combined predicting model based on neural network of variable weighting modulus to rotary sets", China Mechanical Engineering, vol. 14, no. 4, (2003), pp.332-335.

[11] S. Y. Zang, X. Liang and Z. K. Feng, "Establishment of the changeable weight combination forecasting model and its application in regional ecological risk forecasting", Journal of Beijing Forestry University, vol. 29, no. 2, (2007), pp. 203-208.

[12] D.J. Fang and W.B. Weng, "Sales Forecasting System for Chinese Tobacco Wholesalers", Procedia Environmental Sciences, vol.11, (2011), pp.380-386.

[13] B. Luo, L. Wan and W.W. Yan, "A New Type of Combination Forecasting Method Based on PLSThe Application of It in Cigarette Sales Forecasting", American Journal of Operations Research, vol.2 , no.3, (2012), pp.408-416.

[14] L. Wan, B. Luo, H. M. Ji and W. W. Yan, "A type of combination forecasting method based on time series method and PLS", American Journal of Operations Research, vol. 2, no. 4, (2012), pp. 467-472.

[15]X. W. Tang, "Application of error correction models of combination forecasting", Journal of Management Sciences, vol. 5, no. 6, (2002),pp. 53-64. 

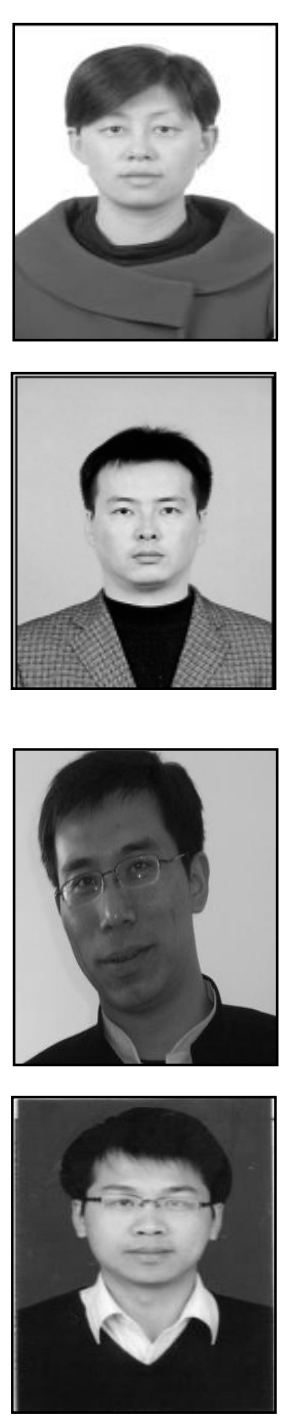

\section{Authors}

Lihua Yang, is a Lecturer, School of Economics and Management, Hubei University of Automotive Technology. She received her B.S degree from Yangtze University in 2003, and her M.S. degree from Southwest Jiao tong University in 2006.Her research interests include data mining and decision support.

Baolin Li, is a Lecturer, School of Economics and Management, Hubei University of Automotive Technology. He received his B.S degree from Lanzhou University in 2005 and his M.S. degree from Northwestern Polytechnic University in 2012. His research interests include data analysis and data management.

Xuetao Li, is a Lecturer, School of Economics and Management, Hubei University of Automotive Technology. He received his M.S. degree from SouthCenter University for Nationalities in 2005. His re search interests include data analysis and financial management.

Lvjiang Yin, is a Lecturer, School of Economics and Managemet, Hubei University f Automotive Technology. He received his B.S de gree from Hubei University of Automotive Technoloyg in 2005, and his M.S. degree from Wuhan University of Science and Technology in 2007. His research interests include intelligence algorithm. 
International Journal of Database Theory and Application Vol.8, No.2 (2015) 